Method 39 ANA/SMA ${ }^{+}$patients $(23$ females; median age: 13.9 years) and 16 healthy subjects (HS, 12 females; median age: 29.5 years) were studied. Cell phenotype was determined by cytofluorimetry using monoclonal antibodies to CD4, CD25 and TIM3. The frequency of cells positive for T-bet, GATA3 and RORC, transcription factors defining Th1, Th2 and Th17 cell subsets, was determined by intracellular staining. Proliferation of $\mathrm{CD}_{2} 5^{-}$, $\mathrm{CD} 25^{-}$Tim $-3^{+}$and $\mathrm{CD} 25^{-}$Tim $-3^{-}$target cells was assessed by ${ }^{3} \mathrm{H}$-thymidine incorporation after 5 -day co-culture with T-regs.

Results The frequency of Tim- $3^{+}$cells within the Th1 and the Th17 subsets was lower in AIH than in HS (Th1: $5.1 \pm 0.7$ vs $13.6 \pm 1.7$, $p<0.001$; Th17: $3.9 \pm 0.3$ vs $8.2 \pm 1.1, p=0.02)$, while there was no difference for Th2 cells. In $\mathrm{AIH}$, there was a negative correlation between the frequency of Tim $-3^{+}$cells and AST levels $(r=-0.47$, $p=0.002$ ) and a positive correlation between the frequency of T-bet ${ }^{+}$ cells and AST levels $(r=0.69, p<0.01)$. Addition of $T$-regs reduced cell proliferation by $26 \%(p=N S)$ in AIH and by $53 \%(p=0.007)$ in HS when undivided CD25 cells were used as targets; by $23 \%$ $(\mathrm{p}=\mathrm{NS})$ and by $25 \%(\mathrm{p}=\mathrm{NS})$ when Tim $-3^{-}$cells were the targets and by $47 \%(p=0.03)$ and by $62 \%(p=0.001)$ when the targets were Tim $-3^{+}$cells.

Conclusion In AIH, reduced frequency of Tim- 3 within the Th1 and Th17 effector cell subsets renders them less prone to T-reg control, CD4 effector cell proliferation depending on the expression of Tim3. T-bet expression characterises effector T-cells likely to mediate liver damage in AIH.

\section{P30 LUNG INJURY AND ITS PROGNOSTIC SIGNIFICANCE IN ACUTE LIVER FAILURE}

doi:10.1136/gutjnl-2011-300857a.30

${ }^{1} \mathrm{~V}$ K Audimoolam, ${ }^{1}{ }^{2} \mathrm{M}$ McPhail, 'S Desai, ${ }^{1} \mathrm{C}$ Willars, ${ }^{1} \mathrm{~W}$ Bernal, ${ }^{1} \mathrm{~J}$ A Wendon, ${ }^{1} \mathrm{G}$ Auzinger. ${ }^{1}$ King's College Hospital; ${ }^{2}$ Imperial College London

Introduction Acute liver failure (ALF) is a multi system illness. Data on the incidence and outcome of acute lung injury (ALI) and acute respiratory distress syndrome (ARDS) complicating ALF is scant ${ }^{1}$. We analysed radiological, gas exchange and ventilator data of consecutive patients admitted with ALF/subacute liver failure (SALF) to a tertiary liver intensive therapy unit (LITU).

Aim The main objective of this assessment was to identify the incidence of ALI/ARDS in ALF/SALF and the impact it has on outcome.

Method All patients with ALF/SALF receiving mechanical ventilation who were admitted between January 2007 and February 2011 were included. Patients were categorised according to the ARDS network consensus definition as: No lung injury present (NALI), ALI (P/F <300 mm Hg) and ARDS (P/F <200 mm Hg). Chest radiographs were independently assessed by two observers for the presence of bilateral infiltrates. Absence of left atrial pressure elevation was based on haemodynamic and echocardiographic assessment.

Results 146 (M: F 71:75) patients with ALF/SALF were studied. 31 patients (21\%) fulfilled ARDS network criteria, 14 ARDS (9.6\%), 17 ALI (11.6\%), within the first $72 \mathrm{~h}$ following LITU admission. ARDS patients required higher levels of positive end expiratory pressure (10 vs $6 \mathrm{ALI}$ and $7 \mathrm{cmH}_{2} \mathrm{O}$ NALI, $\left.\mathrm{p}=0.016\right)$ and had a worse oxygenation index (10.7 vs 4.3 ALI, 4.5 NALI, $p \leq 0.001)$. There was a trend towards reduced compliance of the respiratory system in ARDS and ALI patients ( $p=0.07$ vs NALI) and an increased number of ventilator days (NALI 10d, ALI 12d, ARDS 17d). Duration of LITU stay $(p=0.175)$ and survival $(p=0.877)$ were not affected by the presence of ALI/ARDS. Type of liver disease presentation ALF/SALF, poor prognostic markers of liver failure, that is, lactate, INR, bilirubin, presence of encephalopathy and intracranial hypertension did not correlate with lung injury presentation. Also no association with inotrope requirements $(p=0.495)$, need for extracorporeal renal support $(p=0.565)$ and severity of organ failure scores was found.

Conclusion The incidence of lung injury is relatively low in ALF of mixed aetiology. Less than $10 \%$ of patients fulfilled ARDS criteria. Overall presence of ALI/ARDS appeared to have a limited impact on outcome.

\section{REFERENCE}

1. Baudouin SV, et al. ALI in FHF following paracetamol poisoning. Thorax 1995; 50:399-402.

\section{P31 PREDICTORS OF FLUID RESPONSIVENESS IN PATIENTS WITH ACUTE LIVER FAILURE}

doi:10.1136/gutjnl-2011-300857a.31

${ }^{1} \mathrm{~V}$ K Audimoolam, ${ }^{12} \mathrm{M}$ Mcphail, ${ }^{1} \mathrm{C}$ Willars, ${ }^{1} \mathrm{~W}$ Bernal, ${ }^{1} \mathrm{~J}$ A Wendon, ${ }^{1} \mathrm{G}$ Auzinger. ${ }^{1}$ King's College Hospital, London, UK; ${ }^{2}$ Imperial College, London, UK

Introduction Profound haemodynamic changes are invariably seen in ALF and resemble those found in later stages of septic shock. Vasopressor support is frequently required and in discriminatory fluid resuscitation can worsen intracranial hypertension (ICH) and lung injury. Markers of preload dependency have thus far not been studied in this patient group and response to dynamic manoeuvres such as passive leg raising or end expiratory hold cannot be considered safe in this population due to the high incidence of $\mathrm{ICH}$. Method Patients admitted to a tertiary referral specialist ICU with ALF. All patients were in vasoplegic shock, requiring multiorgan support including controlled mechanical ventilation. Cardiac output monitoring via transpulmonary thermodilution (TPTD) and pulse contour analysis (PiCCO, Pulsion Munich) was performed. Markers of fluid responsiveness were compared between responders $(\mathrm{CI} \geq 15 \%)$ and non-responders to a colloid fluid challenge $(5 \mathrm{ml} / \mathrm{kg}$ IBW). All patients had a transthoracic echocardiogram (TTE) performed before and after fluid administration. The predictive capacity of stroke volume-pulse pressure variation (SVV, PPV) and respiratory change in peak aortic velocity (DV peak) for preload dependency was analysed.

Results 26 patients (mean age 40 (13), 15 M: 11 F,) with mixed aetiology ALF were assessed. The mean APACHE II score was 23 (4) and mean SOFA 15 (2). Change in CI and SVI were closely correlated $(R=0.726, p<0.001)$. There was no difference between those defined as responders using a cut-off of CI or SVI of $10 \%$. When using 15\%, 7 patients would have been classified differently. Intraclass correlation coefficient (ICC) for CI and SVI change was 0.83 (0.62-0.92), confirmed using Pasing \& Blakock regression $(A=$ $-0.278,-0.88$ to $0.16, \mathrm{~B}=1.26,0.88$ to 1.72 ) suggesting haemodynamic changes in both measures are interchangeable. Using a cut-off of a change in CI of $15 \%$ only PPV predicted fluid responsiveness (AUROC $0.79,0.58-0.93, p=0.005$, cut-off $>9 \%$, sensitivity $75 \%$, specificity $62 \%$ ). SVV weakly predicted fluid responsiveness in this cohort (AUROC $0.73,0.52-0.87, p=0.005$, cut-off $>11 \%$ ), While there was a trend towards reduction in DV peak (mean difference $-3 \%, p=0.080)$ this was not different between those defined as fluid responders by CI (Repeated measures ANOVA $p=0.124$ ) and AVV prior to fluid bolus did not predict a CI response (AUROC 0.637, 0.413-825, $\mathrm{p}=0.322$ )

Conclusion Baseline PiCCO parameters predict fluid responsiveness but the respiratory variability in DV peak did not predict a $\mathrm{CI}$ response to fluid bolus in this cohort of ALF patients. PPV may be a more suitable PiCCO index for assessing fluid requirements in patients with ALF than SVV. 